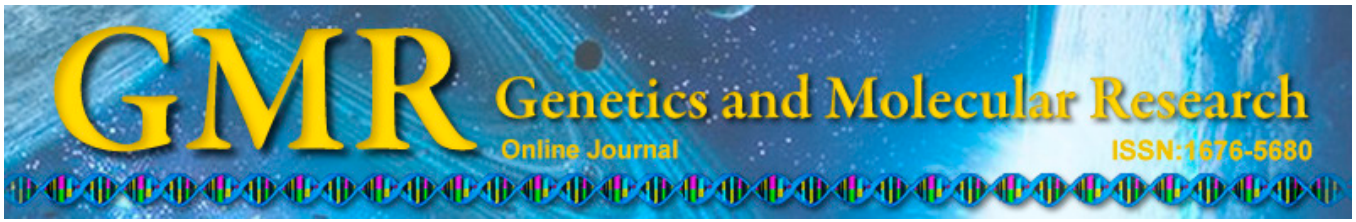

\title{
Immunohistochemistry of lymphocytes in benign lymphoadenosis of oral mucosa
}

\author{
S.-X. Li ${ }^{1 *}$, Q. Li ${ }^{2 *}$, Y.-Q. Yang ${ }^{3}$, L.-J. Jin ${ }^{4}$, Z. Sun ${ }^{1}$ and S.-F. Yu \\ ${ }^{1}$ Department of Periodontics \& Oral Medicine, School of Stomatology, \\ Capital Medical University, Beijing, China \\ ${ }^{2}$ Department of Prosthodontics, School of Stomatology, \\ Capital Medical University, Beijing, China \\ ${ }^{3}$ Department of Orthodontics, School of Stomatology, Hong Kong University, \\ Hong Kong \\ ${ }^{4}$ Department of Periodontics, School of Stomatology, Hong Kong University, \\ Hong Kong \\ ${ }^{5}$ Department of Oral Pathology, School of Stomatology, Peking University, \\ Beijing, China \\ *These authors contributed equally to this study. \\ Corresponding authors: S.-X. Li / Z. Sun \\ E-mail: shuxialicn@163.com / shipingnang@yeah.net
}

Genet. Mol. Res. 14 (2): 7163-7171 (2015)

Received August 14, 2014

Accepted January 21, 2015

Published June 29, 2015

DOI http://dx.doi.org/10.4238/2015.June.29.10

\begin{abstract}
Benign lymphoadenosis of oral mucosa (BLOM) is a common oral mucosa disease and may be regarded as a precancerous lesion. However, the association between its biological behavior and lymphocyte distribution remains unclear. Therefore, to investigate the characteristics of BLOM, we studied the infiltration of lymphocytes associated with it. The expression levels of CD74, CD20, CD3, and $\mathrm{CD} 45 \mathrm{RO}$ were evaluated by immunohistochemical staining in 14 samples from BLOM, 9 samples from BLOM with atypia hyperplasia, 11 samples from BLOM with canceration, and 10 samples from normal oral mucosa tissues. The results were analyzed by two-sample $t$-test using SPSS 10.0 for Windows, and $\mathrm{P}<0.05$ was considered to be sig-
\end{abstract}


nificant. In normal oral mucosa, positive expression levels of CD3 and $\mathrm{CD} 45 \mathrm{RO}$ were presented in the extra-lymphoid follicle, and the expression levels of CD74 and CD20 were negative. In all BLOM groups, the expression level of CD20 was positive except for one case of BLOM with canceration; the expression levels of CD74 were all positive. Positive expression levels of CD3 and CD45RO could be found not only in extra-lymphoid follicles but also in inner-lymphoid follicles in the BLOM groups. The expression levels of CD74 and CD20 in extra-lymphoid follicles, and CD3 and CD45RO in inner-lymphoid follicles in BLOM were significantly higher than in BLOM with canceration. The infiltrated lymphocytes in BLOM comprise T- and B-cells. This indicates that the lymphoid tissue in BLOM is mucosa-associated lymphoid tissue and BLOM is a proliferative lesion.

Key words: CD20; CD74; CD3; CD45RO; Immunohistochemistry; Benign lymphoadenosis of oral mucosa (BLOM)

\section{INTRODUCTION}

Benign lymphoadenosis of oral mucosa (BLOM) is a common disease of the oral mucosa that usually occurs in the lips, cheeks, palate, tongue, and gingiva. Sometimes BLOM may develop in all of the oral mucosa and accompany other oral diseases. The typical pathological appearance of the disease is characterized by follicle-like hyperplasia of the subepithelial lymphocytes. In non-Chinese literature, this lymphocyte proliferation has been considered reactive hyperplasia (Harsany et al., 1980; Wright and Dunsworth, 1983; Mopsik et al., 1992; Anjomshoaa et al., 2013). However, in China this kind of disease has been listed separately as having one typical pathological appearance but a wide variety of clinical manifestations, and has been individually named BLOM (Yu, 2003). Ultrastructural changes of this disease are similar to those of leukoplakia. Clinically, BLOM has a malignant trend and it is regarded as a kind of precancerous lesion. It is claimed in some literature that the canceration rate of this disease is as high as about 10\% (Shun et al., 1992). Literature about the mechanisms of canceration has focused on the changes in epithelial cell morphology, while the role of subepithelial connective tissues in the canceration process has not been reported. In fact, the subepithelial connective tissues play a very important role in the canceration process of diseases, especially in the proliferation of inflammatory cells in the chronic inflammatory diseases, and have a close relationship with disease canceration (Aromando et al., 2008; López-Blanc et al., 2009). As far as we know, few reports have mentioned BLOM, and the association between biological behavior and lymphocyte distribution in BLOM remains unknown. This study attempted to understand the types of lymphocyte proliferation in BLOM, analyze the relationships between different lymphocyte types and epithelial dysplasia, even canceration, and investigate the possible mechanisms. Therefore, to investigate the characteristics of BLOM, we used immunohistochemistry to study the expression levels of CD74, CD20, CD3, and CD45RO of lymphocytes in normal oral mucosa, BLOM, BLOM with atypia hyperplasia, and BLOM with canceration. 


\section{MATERIAL AND METHODS}

\section{Materials}

A total of 44 paraffin-embedded tissue samples were provided by the Pathology Department of the School of Stomatology, Peking University. Of these, 14 were from BLOM, 9 were from BLOM with atypia hyperplasia, 11 were from BLOM with canceration, and 10 were from normal oral mucosa around the neoplasm. This study was conducted in accordance with the Declaration of Helsinki and with approval from the Ethics Committee of Peking University. Written informed consent was obtained from all participants. All the immunohistochemical primary antibodies were mouse anti-human monoclonal antibodies, and the antibodies and immunohistochemical kits were purchased from the Maxim Biotechnology Co., Ltd. (Fuzhou, Fujian, China).

\section{Immunohistochemistry}

Five-micrometer thick sections were dewaxed with xylene and rehydrated in graded ethanol. Endogenous peroxidase activity was blocked by immersion of slides in 3\% hydrogen peroxide for $10 \mathrm{~min}$. To retrieve antigenicity, these sections were then heated in a pressure cooker in $10 \mathrm{mM}$ citrate buffer ( $\mathrm{pH} \mathrm{6}$, for $90 \mathrm{~s}$ after the water boiled). After washing in phosphatebuffered saline, these sections were incubated in normal goat serum for $10 \mathrm{~min}$, then incubated for $1 \mathrm{~h}$ at room temperature with CD20 (1:100) or CD45RO (1:100). Reaction was visualized with a streptavidin-biotin-immunoperoxidase system using 3,3'-diaminobenzidine as chromogen. All sections were then counterstained with hematoxylin.

\section{Quality control}

Positively stained CD74, CD20, CD3, and CD45RO were regarded as positive control groups. For the blank control group, the primary antibodies were replaced with phosphate-buffered saline. Positive spots of CD74, CD20, CD3, and CD45RO were located in the cellular membrane.

\section{Assessment of results}

We chose 10 fields of view near the basal membrane in non-tumor tissues but near the nest cancer in tumor tissues at 400X magnification. The percentage of positive cells in 1000 cells was calculated. The positive lymphocytes in lymphoid follicles or extra-lymphoid follicles were counted.

\section{Statistical analysis}

All data were analyzed by two-sample $t$-test using SPSS 10.0. A P-value of $<0.05$ was considered to be statistically significant.

\section{RESULTS}

CD20 antigen, which was stained brown in the cellular membrane, was expressed in the lymphoid follicles (Figure 1). 

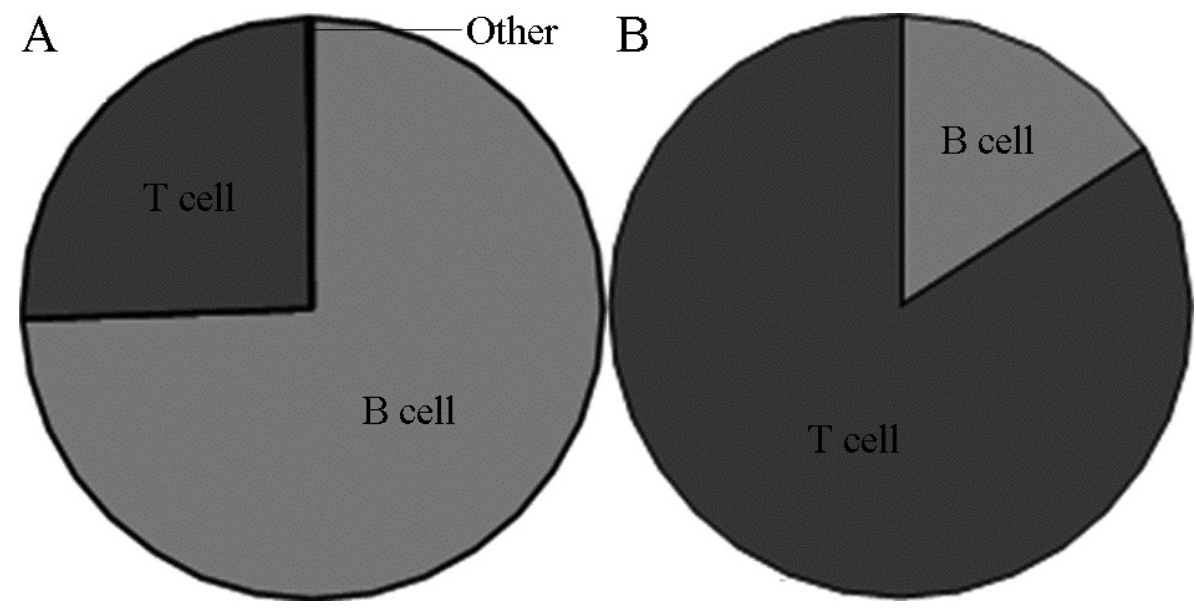

Figure 1. Distribution of infiltrated lymphocytes in BLOM-HE. A. Distribution of infiltrated lymphocytes of innerlymphoid follicle of BLOM. B. Distribution of infiltrated lymphocytes of extra-lymphoid follicle in BLOM.

The staining made the morphology of the lymphoid follicles clear, including their clear borders, their unequal sizes, and the large distances separating them. Therefore, lymphoid follicles were easy to discern by CD20 staining (Figure 2).

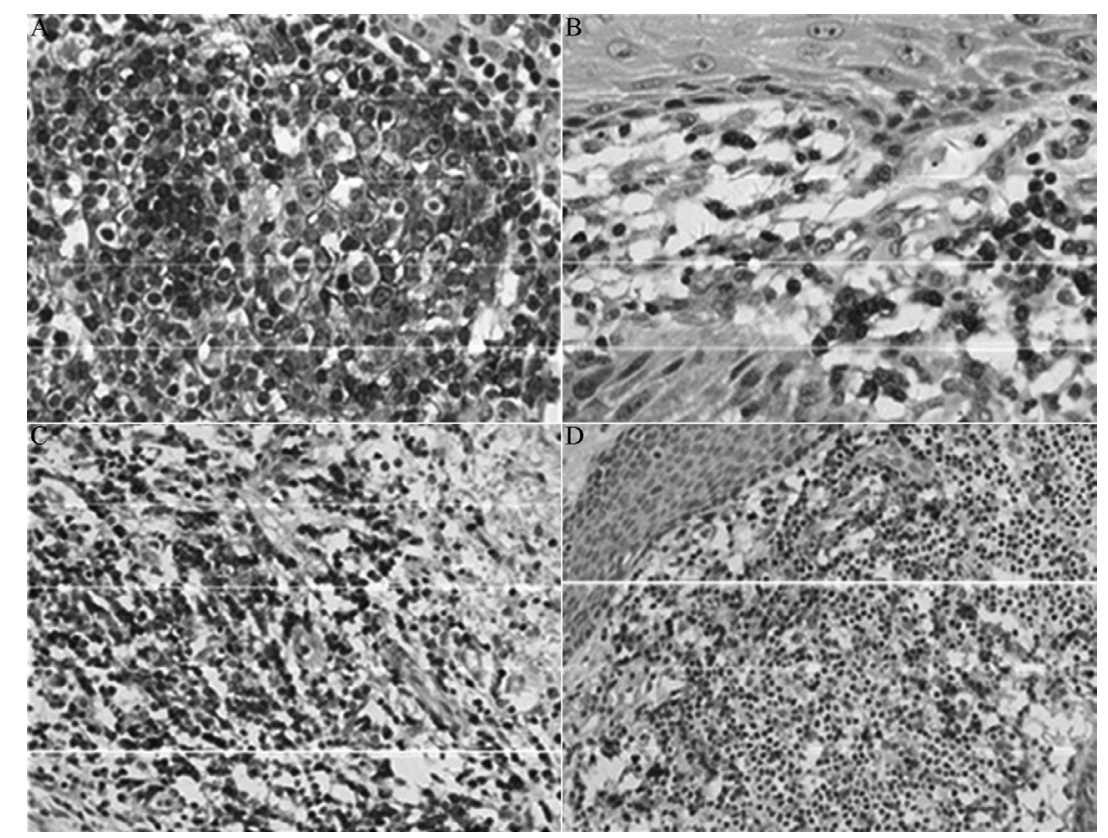

Figure 2. Distribution of infiltrated lymphocytes in BLOM-IHC. A. Expression of CD20 in lymphoid follicles IHC X400; B. expression of CD45RO in lamina propria mucosae IHC X400; C. positive CD45RO cells accumulated in germinal center IHC X200; D. positive CD45RO cells distributed dispersedly in germinal center IHC 200. 
The expression of CD74 was similar to CD20. The lymphocytes with CD3 and CD45RO expression, also stained brown in the cellular membrane, were distributed primarily in the lamina propria mucosa (Figure 2B).

In the normal mucosa group, there were 8 cases of CD45RO positive expression and 10 cases of $\mathrm{CD} 3$ positive expression, in which the staining intensity and the number of positive cells were different. The expression of CD20 was negative and there was 1 case of CD74 positive expression.

In all the BLOM groups, the expression of CD20 was positive except for 1 case in BLOM with canceration. The expression levels of CD3, CD74, and CD45RO were all positive, and the distribution of positive $\mathrm{CD} 3$ and $\mathrm{CD} 45 \mathrm{RO}$ expression was obviously different compared with the normal group; a few instances of positive expression of CD3 and CD45RO occurred in the lymphoid follicle in the BLOM groups.

In all the BLOM groups, CD74, CD20, CD3, and CD45RO expression was consistent with cell distribution. The expression of CD74 and CD20 was mainly observed in the lymphoid follicles, while seldom found in the peripheral tissues; whereas the expression of CD3 and CD45RO occurred in the lamina propria, while a few positive cells were found in the lymphoid follicles. The distribution of cells with positive CD45RO expression in lymphoid follicles took two forms: 1) the positive cells accumulated in the germinal center (Figure 2C); and 2) the positive cells were distributed dispersedly in the germinal center without obvious accumulation, and their density decreased markedly (Figure 2D). The expression of CD3 was not subject to this phenomenon. The expression levels of CD74 and CD20 in extra-lymphoid follicles, and CD3 and CD45RO in inner-lymphoid follicles in BLOM were significantly higher than in BLOM with canceration. (Data are presented in Tables 1 and 2, and the distribution of lymphocytes is shown in Figure 1).

Table 1. Positive rates of CD74, CD20, CD3, and CD45RO expression of inner-lymphoid follicles in different groups (means $\pm \mathrm{SD}, \%$ ).

\begin{tabular}{lcccc}
\hline Groups & CD74 & CD20 & CD3 & CD45RO \\
\hline Normal & & 0 & & 0 \\
BLOM & $76.14 \pm 8.80$ & $74.33 \pm 10.56$ & $25.14 \pm 5.29^{*}$ & $25.42 \pm 10.56^{*}$ \\
BLOM (Dys) & $78.44 \pm 6.78$ & $80.29 \pm 12.00$ & $16.33 \pm 2.45^{*}$ & $16.43 \pm 13.54$ \\
BLOM (Can) & $80.09 \pm 6.27$ & $79.66 \pm 30.67$ & $9.00 \pm 2.57$ & $9.40 \pm 11.56$ \\
\hline
\end{tabular}

$\mathrm{BLOM}(\mathrm{Dys})=\mathrm{BLOM}$ with atypia hyperplasia; BLOM $(\mathrm{Can})=\mathrm{BLOM}$ with canceration; $* \mathrm{P}<0.05$ vs $\mathrm{BLOM}$ with canceration.

Table 2. Positive rates of CD74, CD20, CD3, and CD45RO of extra-lymphoid follicle in different groups (means $\pm \mathrm{SD}, \%$ ).

\begin{tabular}{lcccc}
\hline Groups & CD74 & CD20 & CD3 & CD45RO \\
\hline Normal & 0 & 0 & $90.70 \pm 5.38$ & $89.73 \pm 7.52$ \\
BLOM & $15.36 \pm 3.71^{*}$ & $15.80 \pm 8.08^{*}$ & $83.07 \pm 4.31^{*}$ & $84.51 \pm 7.77$ \\
BLOM (Dys) & $9.44 \pm 2.70$ & $9.02 \pm 7.36$ & $87.33 \pm 6.73$ & $88.82 \pm 7.74$ \\
BLOM (Can) & $9.18 \pm 2.48$ & $9.00 \pm 5.66$ & $88.73 \pm 5.41$ & $87.92 \pm 7.46$ \\
\hline
\end{tabular}

BLOM $($ Dys $)=$ BLOM with atypia hyperplasia; BLOM $($ Can $)=$ BLOM with canceration; $* \mathrm{P}<0.05$ vs BLOM with canceration. 


\section{DISCUSSION}

CD74 and CD20, and CD3 and CD45RO are key markers of B- and T-lymphocytes, respectively. The former (CD74 and CD20) reveal the morphology and distribution of lymphoid follicles; the later (CD3 and CD45RO) demonstrate the distribution and density of T-lymphocytes in lymphoid follicles.

Generally, lymphocytes are classified into two groups: T-lymphocytes are correlated with cellular immunologic response, and B-lymphocytes are correlated with humoral immunological response. Both groups are differentiated from hematopoietic stem cells in the embryo saccus vitellinus and are distributed in peripheral lymphoid organs such as the spleen, lymphoid nodes, and gut-associated lymphoid tissue. As far as we know, there is no lymphatic tissue in the human gastrointestinal tract or other mucosa except for the Peyer's node of the terminal ileum. After infection or repeated stimulation, the autoimmune system forms acquired lymphoid tissues. Therefore, the lymphocytes arising from various kinds of oral mucosa diseases are denominated as acquired lymphoid tissues of the extra-lymphoid node or mucosa-associated lymphoid tissue (MALT) (O’Malley et al., 2014; Orita et al., 2014; Zullo et al., 2014).

Regarding lymphocyte infiltration in oral lichen planus (OLP), Yang et al. (1995) found, using immunohistochemistry, that $98.83 \%$ of cells were T-lymphocytes, whereas $1.16 \%$ were B-lymphocytes. Other studies have reported similar results: T-lymphocytes in the infiltrated lymphocytes of OLP were preponderant and few B-lymphocytes existed (Yang et al., 1995; Liu et al., 2011; Mattila et al., 2011). Cell-mediated immunity plays a key role in the lesion. This indicates that the number of T-lymphocytes has a direct correlation with proliferation of epithelial rate pegs and lesions of the basement membrane. Therefore, the researchers suggested that cell-mediated immunity plays an important role in OLP accompanying participation of humoral immunity. Nie et al. (1997) and Sun et al. (2013) investigated the number of infiltrated lymphocytes in normal oral mucosa, abnormal hyperplasia mucosa, and oral carcinoma. They found that the number of infiltrated lymphocytes increases with aggravation of the disease and reaches a peak at the stage of severe atypia hyperplasia. After that, the lymphocytes decrease. Cheng and Chen (1998) studied BLOM using immunohistochemistry and found that most of the infiltrated inflammation cells, which are located at the lamina propria mucosa, are small lymphocytes and the rest are monocytes and eosinophil granulocytes. Because B-lymphocytes are the main cells in the lymphoid follicle and infiltrated inflammation cells, the authors considered that BLOM should be regarded as a kind of proliferation disease of the regional humoral immune reaction mediated by B-lymphocytes. However, Yu et al. (1991) found that the positive rate of immunoglobulin was only $24 \%$ while the rate of fibrinogen was $60 \%$ by the direct immunofluorescence method. This result does not support the idea that BLOM is an autoimmune disease. In our study, the results for BLOM, similar to those for OLP, indicate that T-lymphocytes are the dominant infiltrated lymphocytes in the lamina propria mucosa $(84.51 \%)$ and B-lymphocytes are in the minority $(15.80 \%)$. The T-lymphocytes in the lamina propria mucosa showed a diffuse distribution. Therefore, we consider that T-lymphocyte-mediated immune response plays an important role in BLOM.

A lymphoid follicle is formed by antigen stimulation. The formation of the germinal center of the lymphoid follicle is a primary process of the humoral immune response (Guan, 1989; Peng, 1991; Gu et al., 1993; Zhang, 1993; International Nasopharynx Cancer Study Group, 1996; Nava and Torres-Cabala, 2013). Some studies have reported increased numbers of Foxp3-positive regulatory T-cells in gastritis, peptic ulcer, and gastric adenocarcinoma (Cheng 
et al., 2012). T-lymphocytes play a key role in the formation of the germinal center, but after that, only a few T-lymphocytes are needed to sustain reaction. The termination of the reaction depends predominantly on the decrease of antigen presented by dendritic cells rather than helper T-lymphocytes (Liang et al., 1995). After reacting with T-lymphocytes stimulated by dendritic cells at the edge of the lymphoid follicles, B-lymphocytes with antigenic specificity accept lymphokines and CD15 from T-lymphocytes and proliferate in the lymphoid follicles. Some of these B-lymphocytes turn into germinal center cells; the others turn into plasmocytes, which can secrete antibodies (Turner and Tiver, 1993). T-lymphocytes play an important role in the formation of the germinal center and the activity of B-lymphocytes. Therefore, T-lymphocytes participate primarily in the reaction of lymphoid follicles and B-lymphocytes are the main cells of lymphoid follicles. To investigate the reaction in the germinal center of lymphoid follicles, we used CD74 and CD20 monoclonal antibodies, and CD3 and CD5RO monoclonal antibodies to mark B-lymphocytes and T-lymphocytes, respectively. The results indicate that the distribution and density of T-lymphocytes in the germinal center have two different types. Xu et al. (2002) investigated the hyperplastic follicles of cervical lymph nodes in laryngeal carcinoma by immunohistochemical staining of CD20 and CD45RO. As with our study, their results showed that the lymphoid follicles are mainly composed of B-lymphocytes but the T-lymphocytes' distribution in it has two main types: the T-lymphocyte-gathering type and the T-lymphocyte-dispersing type. The names derive from the fact that the T-lymphocyte-gathering reaction results in a large number of T-lymphocytes accumulated mainly at the center region of the germinal center. The two types exist in every lymphoid follicle. By counting the number and proportion of follicles that were T-lymphocyte-gathering type, the authors considered that the patient was subject to the T-lymphocyte-gathering type follicle reaction when the proportion of follicles with T-lymphocyte-gathering type exceeded $50 \%$. In contrast, the patient was subject to the T-lymphocytedispersing type follicle reaction when the proportion was less than $50 \%$. By statistical analysis, they suggested that patients with laryngeal carcinoma who exhibited a T-lymphocyte-gathering type follicular response (40.4\%) had a favorable prognosis (5-year survival rate of 68.4\%), but those showing a T-lymphocyte-dispersing type follicular reaction had a bad prognosis (5-year survival rate of 25.0\%) (Xu et al., 2002; Mizuuchi et al., 2014).

$\mathrm{Yu}$ et al. (2013) have shown that approximately $1 / 3$ of breast cancer patients exhibit $\mathrm{CD} 20(+) \mathrm{B}$-cell aggregation and distribution within the stroma, and CD3(+) T-cells gather around the aggregation area of $\mathrm{CD} 20(+)$ B-cells and form a lymphoid follicle-like structure; the $\mathrm{CD} 20(+)$ cell aggregation has prognostic value in hormone receptor-negative breast cancer patients.

In our study, we found three cases of T-lymphocyte-gathering type follicular reaction, two of which were in BLOM and one was in BLOM with epithelial dysplasia. All cases in canceration were T-lymphocyte-dispersing type follicles, which agreed with the findings of $\mathrm{Xu}$ et al. (2002). The expression levels of CD74 and CD20 in extra-lymphoid follicles, and CD3 and CD45RO in inner-lymphoid follicles in BLOM were significantly higher than in BLOM with canceration. However, in our experiment the distribution of $\mathrm{CD} 3(+)$ cells in the lymphoid follicle was not similar to that of CD45RO, and it did not have the two visible types. Therefore, although we cannot be certain, we think that the difference in the T-lymphocytes in lymphoid follicles might be related to the biological behavior of BLOM.

In our previous study, we investigated the pathological characteristics and carcinogenesis mechanism of BLOM and found that the expression of Ki-67 in BLOM with dysplasia and canceration was significantly higher than in BLOM without dysplasia and normal mucosa $(\mathrm{P}<$ 0.05). The microvascular density in BLOM with and without dysplasia and canceration was sig- 
nificantly higher than in normal mucosa $(\mathrm{P}<0.05)$. Apoptosis in $\mathrm{BLOM}$ and oral precancerosis was significantly higher than in oral squamous cell carcinoma and normal mucosa. Following the present study, we compared the two experiments and found that cell proliferation, death, and microvascular density had no conspicuous relation with the distribution of lymphocytes.

To sum up, the lymphocytes of BLOM derive from both T- and B-lymphocytes, so lymphocyte proliferation is polyclonal rather than monoclonal. According to our previous histological study of BLOM, we found there was a large degree of lymphocyte proliferation in the local mucosa and submucosa, and a lymph covering formed by ripe lymphocytes surrounding the germinal center. There were primarily ripe lymphocytes and a large germinal center. We observed karyokinesis but not pathological karyokinesis, and no cell heteromorphism. Therefore, we think BLOM is a disease caused by reactive hyperplasia of lymphocytes. The decrement of T-lymphocytes in lymphoid follicles might be related to the biological behavior of BLOM.

Normally, canceration of a lymphadenism in the digestive tract is a lymphoma, but why is the canceration of oral lymphoadenosis cancer? We attempted to answer that question and concluded that: 1) excepting the long-term cacoethic irritations, there are two types of pathological changes: ulcers, proliferation, and atypia in the epithelium, and MALT in the submucosa; and 2) there is a complex environment in the oral cavity and there are many local irritations. Therefore, the epithelial cells are more susceptible to genetic mutation and cause oral cancer. In other sites in the human body, such as the digestive tract, there are relatively few local irritations of the epithelium but the immune system is always on the alert. The lymphocytes are prone to genetic mutation and cause lymphadenism. However, the definitive explanation requires further study on lymphoadenosis.

\section{Conflicts of interest}

The authors declare no conflict of interest.

\section{ACKNOWLEDGMENTS}

Research supported by the National Natural Science Foundation of China (\#30500559) and the Beijing Municipal Science \& Technology Commission B (\#2005B51).

\section{REFERENCES}

Anjomshoaa I, Bulford LA, Dym H and Woo SB (2013). Florid follicular lymphoid hyperplasia of the hard palatal mucosa managed with intralesional steroids: a case report and review of the literature. J. Oral. Maxillofac. Surg. 71: 1202-1208.

Aromando RF, Pérez MA, Heber EM, Trivillin VA, et al. (2008). Potential role of mast cells in hamster cheek pouch carcinogenesis. Oral. Oncol. 44: 1080-1087.

Cheng B and Chen XH (1998). The clinicopathological and immunohistochemical analysis of benign lymphadenosis of oral mucosa. J. Compr. Stomatol. 14: 99-101.

Cheng HH, Tseng GY, Yang HB, Wang HJ, et al. (2012). Increased numbers of Foxp3-positive regulatory T cells in gastritis, peptic ulcer and gastric adenocarcinoma. World J. Gastroenterol. 18: 34-43.

Gu XZ, Yin WB, Liu TF and Pan GY (1993). Tumor radiotherapeutics. Bei Medical University and Peking Union Medical College Union Publishing House, China, 448-479.

Guan ZZ (1989). Overview of chemotherapy for NPCa. Cancer 8: 120-122.

Harsany DL, Ross J and Fee WE Jr (1980). Follicular lymphoid hyperplasia of the hard palate simulating lymphoma. Otolaryngol. Head Neck Surg. 88: 349-356.

International Nasopharynx Cancer Study Group, VUMCA I Trial (1996). Preliminary results of a randomized trial comparing neoadjuvant chemotherapy (cisplatin, epirubicin, bleomycin) plus radiotherapy $v s$ radiotherapy alone in 
stage IV $(>$ or $=\mathrm{N} 2, \mathrm{M} 0)$ undifferentiated nasopharyngeal carcinoma: a positive effect on progression-free survival. Int. J. Radiat. Oncol. Biol. Phys. 35: 463-469.

Liang JP, Tao ZQ and Wang PZ (1995). Analysis of combined chemotherapy in 48 cases of NPCa (advanced stage) by carboplatin and 5-FU. Cancer 14: 200-201.

Liu GX, Sun JT, Yang MX, Qi XM, et al. (2011). OPN promotes survival of activated T cells by up-regulating CD44 in patients with oral lichen planus. Clin. Immunol. 138: 291-298.

López-Blanc SA, Collet AM, Gandolfo MS, Femopase F, et al. (2009). Nucleolar organizer regions (AgNOR) and subepithelial vascularization as field cancerization markers in oral mucosa biopsies of alcoholic and smoking patients. Oral. Surg. Oral. Med. Oral. Pathol. Oral. Radiol. Endod. 108: 747-753.

Mattila R, Ahlfors E and Syrjänen S (2011). CD27 and CD38 lymphocytes are detected in oral lichen planus lesions. Oral. Surg. Oral. Med. Oral. Pathol. Oral. Radiol. Endod 111: 211-217.

Mizuuchi Y, Aishima S, Hattori M, Ushijima Y, et al. (2014). Follicular pancreatitis, report of a case clinically mimicking pancreatic cancer and literature review. Pathol. Res. Pract. 210: 118-122.

Mopsik ER, Adrian JC and Klein LE (1992). Follicular lymphoid hyperplasia of the hard palate: report of a case. J. Oral. Maxillofac. Surg. 50: 538-540.

Nava VE and Torres-Cabala C (2013). Novel intra-adrenal secondary lymphoid follicle formation. Endocr. Pathol. 24: 248-249.

Nie MH, Chen QM, Li BQ, Zeng XM, et al. (1997). A quantitative pathology study of INF lympholeukocyte during the process of canceration in oral mucosa. West China J. Stomatol. 13: 213-215.

O'Malley DP, Goldstein NS and Banks PM (2014). The recognition and classification of lymphoproliferative disorders of the gut. Hum. Pathol. 45: 899-916.

Orita Y, Sato Y, Kimura N, Marunaka H, et al. (2014). Characteristic ultrasound features of mucosa-associated lymphoid tissue lymphoma of the salivary and thyroid gland. Acta. Otolaryngol. 134: 93-99.

Peng KG (1991). An analysis of therapeutic effect in 223 cases of NPCa (advanced stage) by radiotherapy. Cancer 10: 144-145.

Shun KH, Yu SF and Wu QG (1992). Immunohistochemical study of benign lymphoadenosis of oral mucosa. Chin. J. Stomatol. 27: 104-106.

Sun L, Feng J, Ma L, Liu W, et al. (2013). CD133 expression in oral lichen planus correlated with the risk for progression to oral squamous cell carcinoma. Ann. Diagn Pathol. 17: 486-489.

Turner SL and Tiver KW (1993). Synchronous radiotherapy and chemotherapy in the treatment of nasopharyngeal carcinoma. Int. J. Radiat. Oncol. Biol. Phys. 27: 371-377.

Wright JM and Dunsworth AR (1983). Follicular lymphoid hyperplasia of the hard palate: A benign lymphoproliferative. Oral. Surg. Oral. Med. Oral. Pathol. 55: 162-168.

Xu J, Zhang JW, Ning XM, Xu YD, et al. (2002). Significance of classification of hyperplastic follicular reaction of cervical lymph nodes in laryngeal carcinoma. Chin. J. Clin. Oncol. 9: 613-615.

Yang J, Liang ZM, Wu SC, Xu SM, et al. (1995). An immunohistochemical study of Langerhan's cells in oral lichen planus. West China J. Stomatol. 13: 22-24.

Yu H, Yang J, Jiao S and Wang J (2013). Prognostic value of B lymphocyte infiltration in breast cancer. Nan Fang Yi Ke Da Xие Bao 33: 750-755.

Yu SF (2003). Oral histopathology. 5th edn. People's Publishing House, Beijing, 203-204.

Yu SF, Wei MJ and Pang SZ (1991). The diagnostic value of direct immunofluorescence technic in the diseases of oral mucosa. Chin. J. Stomatol. 26: 112-114.

Zhang L (1993). The chemotherapy of head and neck cancer. Cancer 12: 261-263.

Zullo A, Hassan C, Ridola L, Repici A, et al. (2014). Gastric MALT lymphoma: old and new insights. Ann. Gastroenterol. 27: 27-33. 